Case Report

\title{
Pigmented mucoepidermoid carcinoma, a case report and review of the literature on melanin-pigmented salivary gland tumors
}

\author{
Yasunori Takeda ${ }^{1)}$ and Akira Kurose ${ }^{2)}$ \\ ${ }^{1)}$ Department of Oral Pathology, School of Dentistry, Iwate Medical University, Morioka, Japan \\ ${ }^{2)}$ Department of Pathology, School of Medicine, Iwate Medical University, Morioka, Japan
}

(Received 7 August and accepted 11 September 2006)

\begin{abstract}
This paper reports a well-differentiated mucoepidermoid carcinoma containing numerous melanocytes in the parenchyma in a middle-aged Japanese man. In addition to the characteristic histopathologic features of well-differentiated mucoepidermoid carcinoma, various-formed and -sized, pigmented cells were widely distributed in the parenchyma. Many of these were considered to be melanocytes containing melanin in their cytoplasm. Perusal of the English-language literature revealed only four cases of salivary gland tumors with parenchymal pigmentation: three mucoepidermoid carcinomas and one pleomorphic adenoma. The possible histogenesis of melanocytes in the salivary gland lesions is discussed, although no firm conclusion could be drawn. (J. Oral Sci. 48, 253-256, 2006)
\end{abstract}

Keywords: melanocyte; melanin pigment; mucoepidermoid carcinoma; salivary gland tumor.

\section{Introduction}

Melanocytes are derived from the neural crest. In mammals, during early embryonal life, most melanocyte precursors migrate from the neural crest to the epidermis and hair follicles, while a few reach other locations such

Correspondence to Dr. Yasunori Takeda, Department of Oral Pathology, School of Dentistry, Iwate Medical University, 191 Uchimaru, Morioka 020-8505, Japan

Tel: +81-19-651-5111

Fax: +81-19-621-3321

E-mail: ytakeda@iwate-med.ac.jp as the dermis, sebaceous glands and lactiferous ducts of the nipple, mucous membranes of the upper aerodigestive tract, genitals and anus, orbital cavity, leptomeninges, inner ear, and occasionally certain viscera (1).

It is well known that pre-existing melanocytes are stimulated to produce excess melanin and/or to proliferate to form nevus-like hyperplasia not only in some epithelial tumors of mucocutaneous origin but also in a few extramucocutaneous epithelial tumors. Examples of the former are basal cell carcinoma and squamous cell carcinoma $(2,3)$, hair follicle tumors (4), eccrine gland tumors (5), and mammary and extra-mammary Paget disease $(6,7)$. Examples of the latter, which are rare, are ovarian adenocarcinoma (8), thymic carcinoma $(9,10)$, prostatic carcinoma (11-13), and various types of intra-osseous odontogenic tumors (14).

A review of the literature yielded only four documented cases of salivary gland tumor in which the parenchyma contained melanocytes: three cases of mucoepidermoid carcinoma (15-17), and one of pleomorphic adenoma (18).

The authors have studied the occurrence and distribution of melanocytes in normal salivary glands and salivary gland tumors from Japanese patients, and have discussed their possible histogenesis and pathological significance (18-21). The present paper reports a case of welldifferentiated mucoepidermoid carcinoma in which the parenchyma contained numerous melanocytes.

\section{Case Report}

The patient was a 42-year-old Japanese man who presented with a swelling of the lower lip. He had no past or familial history of any diseases of the head and neck region. The patient stated that the swelling was 
asymptomatic and had been first noted as a tiny painless nodule in the lower lip about 3 years previously, after which it had gradually increased in size. The patient was examined and found to have a well-circumscribed tumor mass, measuring $10 \mathrm{~mm}$ in diameter, on the right side of the lower lip. The tumor was movable, and slightly compressible on palpation. The covering and neighboring mucosa was normal in color. The rest of the physical examination revealed normal intra- and extra-oral findings. A clinical diagnosis of benign minor salivary gland tumor was made, and the lesion was surgically excised along with a clinically normal margin under local anesthesia. The postoperative course was uneventful, and no signs of recurrence have been noted during three years of followup.

\section{Pathological Findings}

The surgical specimen showed the characteristic histological features of a well-differentiated mucoepidermoid carcinoma chiefly composed of mucous secreting cells, non-keratinizing squamous cells, and clear cells (Fig. 1). The carcinoma was well-circumscribed, and variably sized cystic and tubular structures were visible in the parenchyma.

An unusual histological feature was the presence of numerous cells of various forms and sizes with intracytoplasmic brown pigment (Fig. 2). These pigmented cells were distributed throughout approximately two-thirds

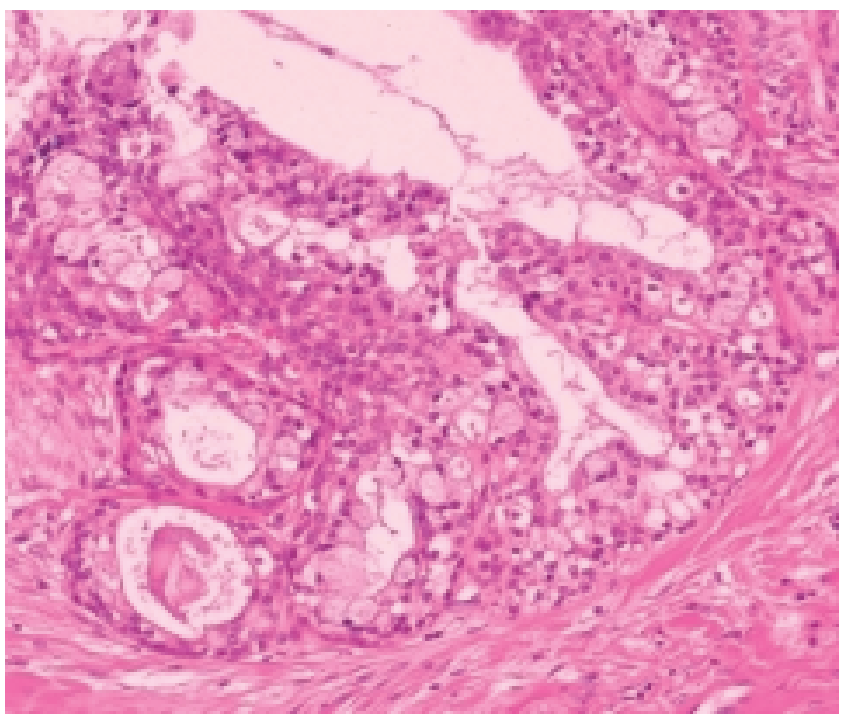

Fig. 1 The tumor, showing the characteristic histological features of well-differentiated mucoepidermoid carcinoma chiefly composed of mucous secreting cells, non-keratinizing squamous cells, and clear cells. Variably sized cystic and tubular structures are visible in the parenchyma $(\mathrm{H}-\mathrm{E} ; \times 200)$. of the parenchyma. Histochemistry indicated that the brown pigment was melanin, with strong positivity with Masson-Fontana's stain for melanin, and bleaching with hydrogen peroxide and potassium permanganate solution. Masson-Fontana's staining vividly demonstrated that the dendritic and spindle shaped pigmented cells were intermingled with tumor cells (Fig. 3). Brown pigment granules were also apparent in the cytoplasm of tumor cells.

The brown pigment was also positive for melanin according to Lillie's ferrous iron method; however, periodic-

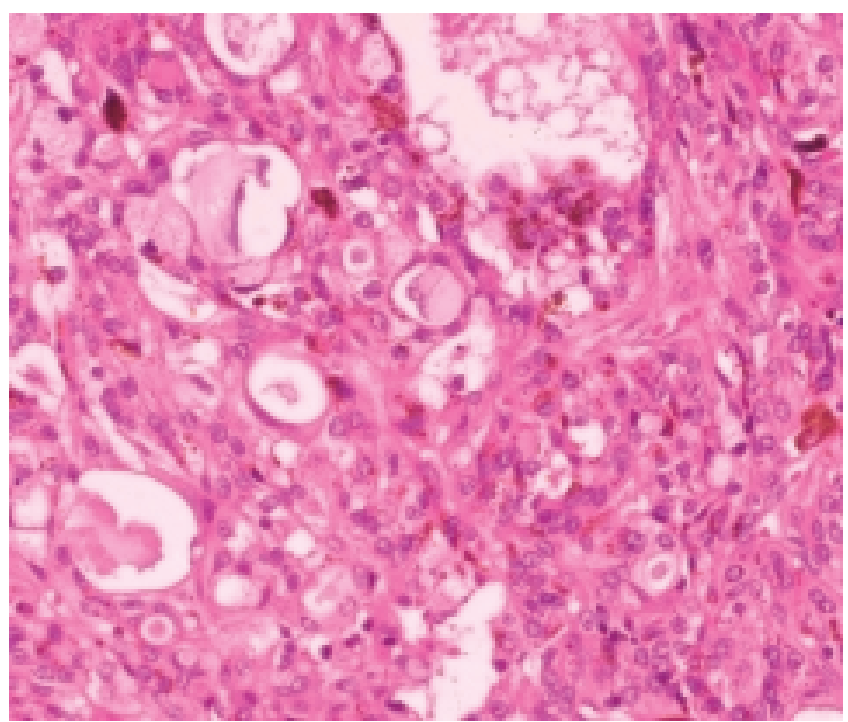

Fig. 2 Numerous, various-formed and-sized cells with intracytoplasmic brown pigment are distributed in the parenchyma $(\mathrm{H}-\mathrm{E} ; \times 400)$.

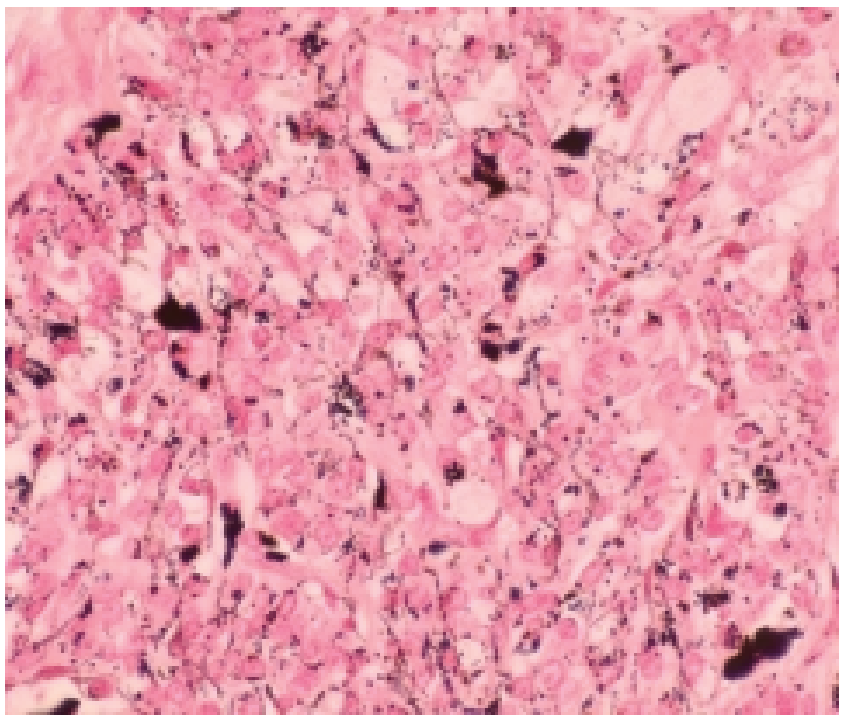

Fig. 3 The pigmented cells distributed in the parenchyma are distinctly positive on staining by Masson-Fontana's silver impregnation method $(\times 400)$. Pigmented, elongated, branching cytoplasmic processes are evident. 
acid-schiff and stains for iron were negative. Immunohistochemistry using bleached sections showed that some of the cells were weakly positive for HMB-45 (1:50 dilution, DAKO, Denmark), and not reactive for vimentin (1:50 dilution, DAKO, Japan).

\section{Discussion}

Only 5 tumors of salivary gland origin containing numerous melanocytes and melanin pigment in the parenchyma and/or stroma have been documented (15-19). Moreover, a review of the literature has yielded no convincing examples of pre-existing melanocytes in the salivary glands. In this regard, we recently published 2 reports documenting the existence of melanocytes in the normal major and minor salivary glands $(20,21)$. In one of these reports (20), routine histopathological examination found melanocytes in the interlobular duct of the parotid gland in only 1 of over 400 autopsy cases. In that case, melanocytes with long cytoplasmic processes were distributed in the basal and suprabasal layers of hyperplastic duct epithelium, and melanin pigment was seen in the duct epithelial cells. In the other report (21), melanocytes in the labial minor salivary glands were found in 8 of 445 autopsies and biopsies (the overall incidence being 1.8\%) using serial sections. Melanocytes were scattered in the periductal and periacinar regions, varied in number, and were frequently seen around the duct system. Such evidence suggests that: (1) inactive melanocytes are occasionally present in salivary glands, similar to those normally seen in clinically non-pigmented oral mucosa, and (2) melanocytes in the salivary glands become activated, proliferate, and produce melanin pigment under certain conditions of tumorigenic transformation of salivary gland epithelia or tumor growth.

Histologically, the present case of well-differentiated mucoepidermoid carcinoma showed that numerous variousshaped and -sized cells with intracytoplasmic brown pigment were intermingled with tumor cells. Histochemical tests, specifically Fontana-Masson's and Lillie's ferrous method, indicated that the intracytoplasmic pigment was melanin. Of these pigmented cells, those with dendritic and spindle appearance were regarded as melanocytes. Immunohistochemically, HMB-45 is a well-known, diagnostically useful antibody that is a specific maker of immature and neoplastic melanocytes in normal tissue and nevus cells (1). However, the spindle and dendritic pigmented cells' lack of strong reactivity with HMB-45 in the present case does not disprove a melanocytic origin for these cells.

Mucoepidermoid carcinoma is a common malignant tumor of both major and minor salivary glands. However, to our knowledge, the present case is only the fourth reported of pigmented mucoepidermoid carcinoma. The first case (15), reported in 1980 in an African, had a labial salivary gland origin, but no detailed histopathologic findings were documented. The second case was in a Caucasian who had been suffering from well-differentiated mucoepidermoid carcinoma of buccal minor salivary gland origin, reported in 1985 (16). Sekine et al. (17) reported a third pigmented mucoepidermoid carcinoma of the palatal minor salivary gland origin in a Japanese patient. Clinical behavior of carcinoma is not related to existence of melanocytes; although one of the reported cases (15) was associated with lymph node metastasis, the others including the present were non-metastatic. Adenoid cystic carcinoma, another common malignant tumor of salivary glands, has not been documented to exhibit parenchymal melanocytes, although we described stromal melanocytosis of an adenoid cystic carcinoma arising from the palatal minor gland (19).

Apart from the distinctive presence of melanocytes and melanin pigment in the tumor parenchyma, it is thought that pigmented cases including the present are histopathologically identical to common mucoepidermoid carcinoma of salivary gland origin. Nonetheless, the presence of melanocytes is a very interesting phenomenon in the field of diagnostic pathology.

\section{Acknowledgments}

This study was supported in part by Grant-in-Aid for High-Tech Research Project (2005-2009) from the Ministry of Education, Culture, Sports, Science and Technology of Japan.

\section{References}

1. Mooi WJ, Krausz T (1992) Biopsy pathology of melanocytic disorders. Chapman \& Hall Medical, London, 1-433

2. Bleehen SS (1975) Pigmented basal cell epithelioma. Light and electron microscopic studies on tumor and cell cultures. Br J Dermatol 93, 361-370

3. James MP, Wells GC, Whimster IW (1978) Spreading pigmented actinic keratoses. Br J Dermatol 98, 373-379

4. Zaim MT (1987) Pilomarticoma with melanocytic hyperplasia: an uncommon occurrence and a diagnostic pitfall. Arch Dermatol 123, 865-866

5. Wilson-Jones E (1971) Pigmented nodular hidradenoma. Arch Dermatol 104, 117-123

6. Jones RE Jr, Austin C, Ackerman AB (1979) Extramammary Paget's disease. A critical reexamination. Am J Dermatopathol 1, 101-132 
7. Neubecker RD, Bradshaw RP (1961) Mucin, melanin, and glycogen in Paget's disease of the breast. Am J Clin Pathol 36, 49-53

8. Waxman M, Vuletin JC, Rosenblatt P, Herzberg FP (1986) Melanocyte colonization of adenocarcinoma arising in an ovarian dermoid. Histopathology 10, 207-215

9. Ho FCS, Ho JCI (1977) Pigmented carcinoid tumour of the thymus. Histopathology 1, 363-369

10. Lagrange W, Dahm HH, Karstens J, Feichtinger J, Mittermayer C (1987) Melanocytic neuroendocrine carcinoma of the thymus. Cancer 59, 484-488

11. Aguilar M, Gaffney EF, Finnerty DP (1982) Prostatic melanosis with involvement of benign and malignant epithelium. J Urol 128, 825-827

12. Furusato M, Matsumoto I, Kato H, Takahashi H, Kawashima Y, Wada T, Suzuki M, Aizawa S (1989) Prostatic carcinoma with melanosis. Prostate 15, 6569

13. Rios CN, Wright JR (1976) Melanosis of the prostate gland: report of a case with neoplastic epithelium involvement. J Urol 115, 616-617

14. Takeda Y, Sato H, Satoh M, Nakamura S, Yamamoto H (2000) Pigmented ameloblastic fibrodentinoma: a novel melanin-pigmented intraosseous odontogenic lesion. Virchows Arch 437, 454-458

15. Thomas KM, Hutt MSR, Borgstein J (1980) Salivary gland tumors in Malawi. Cancer 46, 2328-2334

16. Aufdemorte TB, van Sickels JE, Glass BJ (1985) Melanin pigmentation in a mucoepidermoid tumor of a minor salivary gland. J Oral Maxillofac Surg 43, 876-879

17. Sekine J, Anami M, Fujita S, Vieth M, Inokuchi T (2005) A case of mucoepidermoid carcinoma with melanin pigmentation manifested in the palate. Virchows Arch 446, 460-462

18. Takeda Y, Satoh M, Nakamura S (2004) Pigmented pleomorphic adenoma, a novel melanin-pigmented benign salivary gland tumor. Virchows Arch 445, 199-202

19. Takeda Y (1996) Stromal melanocytosis of an adenoid cystic carcinoma arising from the palatal minor salivary gland. Pathol Int 46, 467-470

20. Takeda Y (1997) Melanocytes in the human parotid gland. Pathol Int 47, 581-583

21. Takeda Y (2000) Existence and distribution of melanocytes and HMB-45-positive cells in the human minor salivary glands. Pathol Int 50, 15-19 